\title{
VARIAÇÃO SAZONAL DA BIOQUÍMICA SANGUÍNEA EM IGUANAS: ASPECTOS FISIOPATOLÓGICOS E ANÁLISE DE 4 CASOS
}

\author{
Máyra Dias SILVEIRA ${ }^{1 *}$, José Edgard de Oliveira ALVES ${ }^{2}$, Mônica dos Santos BRAGA ${ }^{3}$ \& Ana Paula \\ Martinez de ABREU ${ }^{4}$
}

1 Médica Veterinária Autônoma, Pinheiral, RJ, Brasil.

2 Universidade Estadual do Norte Fluminense, Campos dos Goytacazes, RJ, Brasil.

3 Zoológico Municipal de Volta Redonda, Volta Redonda, RJ, Brasil.

4 Universidade Severino Sombra, Vassouras, RJ, Brasil.

*Autor para correspondência: mayraa_dias@hotmail.com

http://dx.doi.org/10.18571/acbm.107

\section{RESUMO}

Assim como os demais répteis, as iguanas são animais ectotérmicos e o clima e outros fatores ambientais podem exercer forte influência sobre a fisiologia e os parâmetros hematológicos desta espécie e com a crescente introdução destes animais como pets exóticos torna-se grande a necessidade de maiores informações sobre as alterações fisiológicas e patológicas na clínica de répteis. O presente estudo tem como objetivo investigar as variações sazonais de parâmetros bioquímicos de iguanas-verdes (Iguana iguana) criadas em cativeiro na cidade de Volta Redonda, Rio de Janeiro. Foram utilizadas 4 iguanas-verdes adultas, das quais foram colhidas amostras de sangue em janeiro e julho de 2014 e analisadas as concentrações de colesterol, triglicerídeos, glicose, ácido úrico, ureia, cálcio e fósforo. Também foram monitorados os índices meteorológicos de umidade, precipitação e temperatura em ambos os meses. Nossos resultados apontaram aumento na concentração sanguínea de ureia em julho em relação a janeiro $(6,3 \pm 0,70 \mathrm{mg} / \mathrm{dL}$ e $3,8 \pm 1,03$, respectivamente) e aumento na relação Ca:P de $1,08 \pm 0,15$ em janeiro para $2,45 \pm 0,40$ na segunda avaliação. Os demais parâmetros não apresentaram alterações significantes nas comparações entre os meses estudados. Conclui-se que a temperatura, estação do ano, dieta, sexo e as condições de manejo às quais estes animais são submetidos têm importante impacto nos resultados de exames laboratoriais. Espera-se que o conjunto de resultados observados neste trabalho possa servir como referência para outros trabalhos com o mesmo caráter de observação.

Palavras-chave: Hematologia; Répteis; Manejo de animais exóticos.

\begin{abstract}
Seasonal variation of the blood biochemistry of iguana: Pathophysiological features and analysis of 4 cases. As well as other reptiles, the iguanas are ectothermic animals and the weather and others ambiental factors may to exert a strong influence over the physiology and the haematological parameters of this specie and the growing introduction of this animals as exotic pets it becomes great the need for more knowledge about the physiological ant pathological alterations in the reptiles clinic. This study aims to investigate the seasonal variance of biochemistry parameters of green iguanas (Iguana iguana) created in captivity in the city Volta Redonda, state of Rio de Janeiro. Were used 4 green iguanas, of which were gathered blood samples in january and july, 2014, and analysed the concentrations of cholesterol, triglycerides, glucose, uric acid, urea, calcium and phosphorus. Also were monitored the meteorological index of humidity, precipitation and temperature in both months. Our results showed increase on blood concentration of urea in july regarding to january $(6,3 \pm 0,70$ and $3,8 \pm 1,03 \mathrm{mg} / \mathrm{dL}$, respectively) and increase of $\mathrm{Ca}: \mathrm{P}$ reason of $1,08 \pm 0,15$ in january to $2,45 \pm 0,40$ in second assessment. The others parameters does not presented significant alterations on the comparison among the studied months.
\end{abstract}


It concludes that the temperature, season, diet, gender and the management conditions of which these animals are submitted has important impact on results of laboratory exams. It is expected that the set of observed results in this paper may contribute as reference to other studies with the same character of observation.

Keywords: Haematology; Reptiles; Exotic animals management.

\section{Introdução}

A espécie Iguana iguana pertencente à ordem Squamata, subordem Lacertilia, da família Iguanidae e se distribui do México ao Brasil Central e Paraguai (VITT et al., 2008). No Brasil, é encontrada em parte da região Centro-Oeste, Pantanal e na Caatinga (CAMPOS, 2003). Segundo Andrade (2009), os lagartos dessa família se alimentam, quando jovens, principalmente de insetos e na vida adulta são herbívoros generalistas (alimentam-se de folhas, brotos, flores e frutos). Segundo um levantamento coordenado pela Sociedade Brasileira de Herpetologia, até o ano de 2005, eram conhecidas 641 espécies de répteis no território brasileiro, representando cerca de $8 \%$ das mais de oito mil espécies conhecidas no mundo (MARTINS; MOLINA, 2008).

Por serem ectotérmicos, repteis são dependentes das condições ambientais para controlar a temperatura corporal e regular seu metabolismo (ROSENTHAL, 2002). De acordo com Barten (2006), a ecologia térmica dos répteis é complexa e envolve muitos fatores. A manutenção da temperatura corpórea apropriada para as atividades metabólicas se dá pelo equilíbrio entre o comportamento e a fisiologia do indivíduo. Para cada atividade diária que o lagarto realiza, tal qual forrageamento, digestão de alimentos, reprodução ou interações sociais, por exemplo, os processos comportamentais e fisiológicos em cada uma dessas atividades ocorrem sob determinadas temperaturas corpóreas que são fisiologicamente apropriadas para a ocasião (ROCHA et al., 2009). Em cativeiro, répteis estressados, doentes ou feridos devem alcançar suas temperaturas ideais para melhorar as funções do sistema imunológico. A absorção de drogas e sua distribuição são influenciadas pela temperatura e os répteis devem manter sua temperatura ideal quando estiverem sob tratamento medicinal ou procedimentos anestésicos (BARTEN, 2006).

Poucos estudos foram realizados em iguanas-verdes para se determinar como interpretar a patologia clínica nesta espécie. Parâmetros hematológicos e bioquímicos em reptilianos podem variar de acordo com o gênero, idade, estado nutricional, estado fisiológico e pela variação da temperatura e de outros fatores ambientais (CAMPBELL, 2006). Além disso, a necessidade de informações sobre as alterações fisiológicas e patológicas é crescente na clínica de répteis. Nas últimas décadas esses animais vem sendo cada vez mais vistos como pets exóticos e expostos em zoológicos e o conhecimento da ecologia e biologia da espécie é necessário para avaliação da saúde desses indivíduos em cativeiro. Portanto, a carência de estudos expõe a importância de investigações mais aprofundadas, pois os valores hematológicos e bioquímicos normais, mesmo para as espécies mais populares, ainda são de conhecimento insuficiente (DIVERS et al., 1996).

Diante da necessidade de maiores esclarecimentos sobre a maneira como as condições climáticas de diferentes épocas do ano interferem na fisiologia destes animais, o presente estudo tem como objetivo investigar a ocorrência de variações sazonais em parâmetros hematológicos de iguanas-verdes (Iguana iguana) criadas em cativeiro na cidade de Volta Redonda, estado do Rio de Janeiro.

\section{Material e Métodos}

Toda a metodologia foi devidamente aprovada pelo Comitê de Ética no Uso de Animais (CEUA) da Universidade Severino Sombra, sob protocolo número 014/2014. Foram utilizadas quatro iguanas-verdes adultas (Iguana iguana) pertencentes ao plantel de répteis do Zoológico 
Municipal de Volta Redonda, Rio de Janeiro, para avaliação dos parâmetros hematológicos nos períodos de janeiro de 2014 e julho do mesmo ano. Estes animais eram criados em recinto de área retangular, que possuía área de sombra artificial, boa irradiação solar, troncos artificiais aéreos e piso gramado e de alvenaria. A alimentação incluía vegetais folhosos e frutas (oferecidos diariamente) e ração comercial para gatos (oferecida para os animais duas vezes por semana).

Todos os animais foram previamente tratados com antiparasitário a base de febantel e pamoato de pirantel (Drontal Puppy, Bayer S. A., São Paulo, SP, Brasil) por via oral em duas administrações com intervalo de 15 dias entre si, de modo a garantir que apenas animais não parasitados fossem utilizados no estudo. Os animais foram capturados com um puçá, sexados, pesados e medidos. A sexagem foi feita observando-se a presença dos poros femorais e précloacais de cada indivíduo.

Foram colhidas amostras de aproximadamente $1 \mathrm{ml}$ de sangue de cada animal através das veias abdominal e veia caudal ventral, utilizando seringas descartáveis de 1 e $3 \mathrm{ml}$ de $22 \mathrm{G}$. As amostras foram identificadas e armazenadas em frascos plásticos de fundo cônico (Eppendorf®) previamente rinçado com heparina sódica e em tubos a vácuo para coleta de sangue (BD Vacutainer®) com heparina de lítio e transportadas sob refrigeração em caixa de isopor térmica até os laboratórios de Patologia Clínica da Fazenda Experimental da Universidade Severino Sombra e Cambuá Vet Laboratorial, onde o material foi processado para avaliação dos seguintes parâmetros: Ácido úrico, ureia, glicose, triglicerídeos, colesterol, cálcio e fósforo.

Os dados sobre as condições meteorológicas, tais quais os índices de umidade, precipitação atmosférica e temperatura máxima e mínima dos meses de janeiro e julho de 2014, foram retirados do Banco de Dados Meteorológicos para Ensino e Pesquisa (BDMEP) e do Instituto Nacional de Meteorologia (INMET).

Os resultados foram tabulados e expressos como a média \pm o erro padrão da média (E.P.M). As comparações entre os parâmetros avaliados em janeiro e julho foram feitas utilizando o Teste $t$ pareado de Student, considerando significantes os valores de $p$ inferiores a $0,05(p<0,05)$.

\section{Resultados e Discussão}

Das quatro iguanas utilizadas no estudo, três eram do sexo masculino e uma fêmea, com peso médio de 4,25 $\pm 1,31 \mathrm{~kg}$ em janeiro e 4,0 $\pm 0,84 \mathrm{~kg}$ em julho. Estes animais apresentavam comprimentos rostrocloacais entre 0,35 e $0,47 \mathrm{~m}$ e comprimento total, que inclui a mensuração rostrocloacal mais a medida da cauda, entre 1,09 e 1,20 m.

Ao analisarmos os dados das médias referentes às variações climáticas de temperatura e umidade deste trabalho, nota-se que a cidade de Volta Redonda possui uma variação considerável entre os meses estudados, onde se observa que as médias para temperatura máxima e umidade do mês de janeiro extrapolaram os valores de referência para as condições ideais para estes animais, bem como os valores para temperatura mínima e máxima e umidade no mês de julho (tabela 1). Segundo Barten (2006), as faixas de temperatura ótima em cativeiro para iguanas-verdes durante a noite e o dia variam de $19,4{ }^{\circ} \mathrm{C}$ a $32,2^{\circ} \mathrm{C}$, enquanto que a umidade ideal fica em torno de 50 a $70 \%$. Por este motivo é notável o estresse térmico sofrido por esses animais. 
Tabela 1. Índices meteorológicos referentes aos meses de janeiro e julho de 2014 na cidade de Volta Redonda, RJ.

\begin{tabular}{lcccc} 
& \multirow{2}{*}{ PARÂMETRO } & \multicolumn{2}{c}{ JANEIRO } & \multicolumn{2}{c}{ JULHO } \\
\cline { 2 - 5 } & Média & EPM & Média & EPM \\
\hline Umidade $(\%)$ & 72,92 & $\pm 1,89$ & $80,32^{*}$ & $\pm 1,49$ \\
Precipitação $(\mathrm{mm})$ & 4,59 & $\pm 1,60$ & 1,75 & $\pm 1,11$ \\
Temp. Min. $\left({ }^{\circ} \mathrm{C}\right)$ & 19,98 & $\pm 0,30$ & $14,1^{*}$ & $\pm 0,35$ \\
Temp. Máx. $\left({ }^{\circ} \mathrm{C}\right)$ & 33,9 & $\pm 0,23$ & $24,1^{*}$ & $\pm 0,61$ \\
\hline
\end{tabular}

EPM: Erro Padrão da Média. Temp. Min.: Temperatura mínima; Temp. Máx.: Temperatura máxima.

*Parâmetros em que foram observadas diferenças estatísticas $(p<0,05)$, segundo o Test $t$.

Fontes de dados: BDMET e INMET.

A umidade é um fator importante, mas muitas vezes esquecido no manejo de lagartos em cativeiro. Em geral, as espécies de florestas tropicais precisam de umidade maior que espécies de deserto, mas sabe-se que a maioria das espécies sobrevive bem em níveis de umidade entre $50 \%$ a $70 \%$. Embora a associação de uma alta umidade e temperatura pode resultar no rápido crescimento de bactérias e fungos nos recintos, isso pode ser evitado com ventilação adequada. Uma correta umidade do ambiente também é necessária para garantir uma ecdise adequada (BARTEN, 2006).

Tabela 2. Comparação das análises bioquímicas realizadas em janeiro e julho de 2014 em iguanas-verdes criadas em cativeiro.

\begin{tabular}{|c|c|c|c|c|}
\hline \multirow{2}{*}{ PARÂMETRO } & \multicolumn{2}{|c|}{ JANEIRO } & \multicolumn{2}{|c|}{ JULHO } \\
\hline & Média & EPM & Média & EPM \\
\hline Colesterol (mg/dL) & 86,8 & $\pm 14,2$ & 122,5 & $\pm 34,35$ \\
\hline Triglicerídeos (mg/dL) & 474,0 & $\pm 83,73$ & 386,8 & $\pm 154,52$ \\
\hline Glicose (mg/dL) & 255,0 & $\pm 28,88$ & 197,0 & $\pm 33,0$ \\
\hline Ácido Úrico (mg/dL) & 1,2 & $\pm 0,26$ & 2,9 & $\pm 1,68$ \\
\hline Ureia (mg/dL) & 3,8 & $\pm 1,03$ & $6,3^{*}$ & $\pm 0,70$ \\
\hline Cálcio (mg/dL) & 8,2 & $\pm 0,42$ & 12,4 & $\pm 1,12$ \\
\hline Fósforo (mg/dL) & 8,1 & $\pm 1,2$ & 5,7 & $\pm 1,23$ \\
\hline $\mathrm{Ca}: \mathrm{P}$ & 1,08 & $\pm 0,15$ & $2,45^{*}$ & $\pm 0,40$ \\
\hline
\end{tabular}

EPM: Erro Padrão da Média. mg/dL: Miligramas por decilitro. Ca:P: Relação cálcio/fósforo.

*Parâmetros em que foram observadas diferenças estatísticas $(p<0,05)$, segundo o Test $t$.

Não houve diferença estatística $(p=0,5)$ nas comparações entre as médias de colesterol nos períodos estudados e as médias obtidas em ambos os períodos encontram-se dentro da faixa de normalidade para este parâmetro, que pode variar de 82 a $214 \mathrm{mg} / \mathrm{dL}$ para machos e 204 a 347 $\mathrm{mg} / \mathrm{dL}$ para fêmeas (MADER, 2006). Todavia, observou-se hipocolesterolemia em dois animais da população estudada (53 mg/dL em um macho e $82 \mathrm{mg} / \mathrm{dL}$ em uma fêmea) no mês de janeiro e isso pode ter ocorrido devido à ação tireoidiana durante a ecdise (RAMEH-DE-ALBUQUERQUE, 2007), porém não é possível a confirmação desta hipótese, pois não foram observados sinais de troca de pele nestes animais. Já as concentrações médias de triglicerídeos obtidas tanto para o mês de janeiro quanto em julho não apresentaram diferenças nas comparações entre os períodos e em ambos os momentos encontravam-se dentro da faixa de normalidade, entre 53 e $691 \mathrm{mg} / \mathrm{dL}$ (MADER, 2006).

Não foram observadas alterações significantes $(p=0,08)$ nas análises realizadas para os valores médios de glicose comparando-se os períodos avaliados. Segundo Mader (2006), em iguanas machos são considerados normais para este parâmetros valores entre 70 a $244 \mathrm{mg} / \mathrm{dL}$ e em fêmeas de 105 a $258 \mathrm{mg} / \mathrm{dL}$. Ademais, sabe-se que o controle hormonal da glicemia pela insulina e glucagon pancreáticos em répteis é semelhante aos mamíferos e outros vertebrados. A concentração de glicose no sangue de répteis normais também pode variar de acordo com a 
espécie, estado nutricional e as condições ambientais, tal como a temperatura (CAMPBELL, 2006). Segundo Rameh-de-Albuquerque (2007), condições de privação alimentar, má nutrição, dietas com altos níveis proteicos, hepatopatias severas, sepse e doenças endócrinas são situações onde pode se observar hipoglicemia. Por outro lado, a hiperglicemia está relacionada a problemas metabólicos, doenças sistêmicas e variáveis fisiológica, porém, não é um indicador específico de doença pancreática ou diabetes mellitus (SILVESTRE et al., 2013).

Dois animais machos apresentaram valores de ácido úrico $(0,6$ e 1,0 mg/dL) abaixo da faixa de normalidade para o gênero masculino, que pode variar de 1,5 a 5,8 $\mathrm{mg} / \mathrm{dL}$ de ácido úrico na Iguana iguana. Embora estes valores tenham sido observados abaixo dos parâmetros de normalidade, não foi possível encontrar na literatura atual informações que expliquem esta condição em répteis. Entretanto, observou-se um quadro de elevação dos níveis de ácido úrico em um dos animais, subindo de 1,6 mg/dL em janeiro para 7,90 mg/dL em julho. Segundo Almosny e Monteiro (2007), o aumento das concentrações de ácido úrico deve ser avaliado com cautela, pois a elevação deste pode ser decorrente de dietas ricas em proteínas e que somente níveis plasmáticos acima de $15 \mathrm{mg} / \mathrm{dL}$ podem caracterizar hiperuricidemia em répteis, geralmente associada à doença renal. O mesmo animal também apresentou hipocolesterolemia, hipocalemia, hiperglicemia e hipertrigliceridemia. A condição de hipocalemia em repteis pode ocorrer por anorexia, inanição, neoplasias ou deficiências nutricionais (SILVESTRE et al., 2013).

Nota-se que houve significante variação entre as médias de ureia nos meses estudados, onde percebe-se um aumento $(p=0,03)$ dos níveis plasmáticos em julho quando comparados aos valores encontrados em janeiro. Este resultado é corroborado por Silvestre et al. (2013), que afirma que este parâmetro apresenta alta variação sazonal, chegando a valores máximos durante a hibernação de algumas espécies. Apesar desta diferença, em ambos os períodos os valores continuaram dentro dos parâmetros de normalidade para a espécie, que pode variar de 0 a $11 \mathrm{mg} / \mathrm{dL}$, de acordo com Mader (2006).

Os valores normais de cálcio para iguanas-verdes encontrados na literatura variam de 8,6 a $14,0 \mathrm{mg} / \mathrm{dL}$ (MADER, 2006). Observando a tabela 2, percebe-se que houve discreta hipocalcemia na avaliação realizada em janeiro. Em contrapartida, houve discreto aumento na concentração plasmática de fósforo em janeiro, cujos valores considerados normais variam de 3,2 a 9,3 mg/dL, como cita Mader (2006). Todavia, de acordo com o mesmo autor, ambos os parâmetros encontravam-se dentro da faixa de normalidade nas análises laboratóriais realizadas em julho. De acordo com Campbell (2006), a hipocalcemia pode ocorrer devido à deficiência de cálcio e vitamina D3 na dieta ou excesso de ingestão de fósforo na alimentação.

Segundo Divers (2000), a determinação da concentração plasmática de cálcio e fósforo é importante no diagnóstico de várias doenças e os valores de $\mathrm{Ca}: \mathrm{P}$ devem ser maiores ou iguais a $1: 1$. Nossa análise apontou diferença estatística $(p=0,03)$ para a relação Ca:P em julho em relação à primeira avaliação, mas em ambos os momentos estudados estes valores encontravam-se dentro da normalidade. Sabe-se que uma relação de Ca:P menor que 1:1 sugere doença renal (SILVESTRE et al., 2013).

Através da análise da dieta destes animais, bem como do recinto das iguanas submetidas ao presente estudo, podem ser destacados alguns aspectos que também podem contribuir para alterações em exames hematológicos. Notam-se algumas divergências de manejo ao serem levadas em consideração as referências bibliográficas utilizadas (BARTEN, 2002). A oferta de proteína de origem animal na dieta não é cientificamente comprovada como eficaz, considerando que estes animais são naturalmente herbívoros. Consequentemente, o consumo exacerbado de proteína pode causar alterações nos exames laboratoriais bioquímicos. Ainda, a convivência de répteis em um mesmo recinto pode gerar competição pela comida, haja vista que estes animais são territorialistas. No recinto, todos os animais se encontravam em um sistema de criação de regime extensivo. Os animais não estavam em um ambiente controlado, passível de alterações climáticas como temperatura, vento e chuva, o que é a realidade constatada na maioria dos criatórios e zoológicos 
do país, tendo em vista as dificuldades estruturais para manutenção destes animais em ambientes controlados e individuais.

\section{Conclusão}

Ao trabalharmos com exames laboratoriais de animais ectotérmicos, como as iguanas, devem ser levados sempre em consideração as variações de temperatura, estação do ano, sexo e condições de manejo destes animais. Indivíduos não confinados, onde o ambiente não pode ser controlado, não estão bem protegidos de mudanças e variações de temperatura, umidade e insolação e isto pode afetar os resultados das amostras. A dieta é fator importante na interpretação dos dados bioquímicos, uma vez que eventuais alterações e desequilíbrios podem estar associados ao manejo inadequado.

Estudos em populações maiores de Iguana iguana e com observações em períodos mais prolongados ainda são necessários para que se alcance uma melhor compreensão sobre a relação de fatores climáticos com o metabolismo e a utilização destes dados com maior precisão na clínica da espécie estudada. No entanto, espera-se que o conjunto de resultados observados em nosso estudo, possa servir como referência para futuros trabalhos com o mesmo caráter de observação.

\section{Agradecimentos}

Zoológico Municipal de Volta Redonda, Universidade Severino Sombra e Cambuá Vet Laboratorial.

\section{Referências}

ALMOSNY, N. R. P.; MONTEIRO, A. M. Patologia Clínica. In: CUBAS, Z.S.; SILVA, J. C. R.; CATÃO-DIAS, J. L. (Orgs.). Tratado de Animais Selvagens: Medicina Veterinária. São Paulo: Roca, 2007. p. 939-367.

ANDRADE, C. A. F. Iguana verde (Iguana iguana). Universidade Federal de Viçosa: MZUFV, Bicho da Vez, n. 6, p. 1-3, 2009.

BARTEN, S. L. Criação e manejo do iguana verde. In: Bayer Exotics Symposium: Selected Papers on the Green Iguana and Antimicrobials in Exotic Pets, p. 13-22, 2002.

BARTEN, S. L. Lizards. In: MADER, D. R. (Ed.). Reptile Medicine and Surgery. 2nd ed. St. Louis: Sauders-Elsevier, 2006, p. 59-77.

CAMPBELL, T. W. Clinical pathology of Reptiles. In: MADER, D. R. (Ed.). Reptile Medicine and Surgery. 2nd ed. St. Louis: Sauders-Elsevier, 2006, p. 453-470.

CAMPOS, Z. M. S. Biologia reprodutiva de Iguana no rio Paraguai. Pantanal, Brasil. Corumbá: Embrapa - Comunicado Técnico nº. 30, ISSN 1517-4875, 2003. 3 p.

DIVERS, S. J.; REDMAYNE, G.; AVES, K. Haematological and biochemical of 10 green iguanas (Iguana iguana). The Veterinary Record, London, v. 138, n. 3, p. 203-205, 1996.

DIVERS, S. J. Reptilian Renal and Reproductive Disease Diagnosis. In: FUDGE, A.M. Laboratory Medicine - Avian and Exotic Pets. Philadelphia: Saunders, 2000, p.217-222.

MADER, D. R. Reptile Medicine and Surgery. 2nd ed. St. Louis: Sauders-Elsevier, 2006, 1264 p. 
MARTINS, M. R. C.; MOLINA, F. B. Panorama Geral dos Répteis Ameaçados do Brasil. In: MACHADO, A. B. M.; DRUMMOND, G. M.; PAGLIA, A. P (Eds.). Livro Vermelho da Fauna Brasileira Ameaçada de Extinção. Brasília: MMA; Belo Horizonte: Ed. Fundação Biodiversitas, 2008, 1420 p.

RAMEH-DE-ALBUQUERQUE, L. C. Aspectos Hematológicos, Bioquímicos, Morfológicos e Citoquímicos de Células Sanguíneas em Viperídeos Neotropicais dos Gêneros Bothrops e Crotalus mantidos em cativeiro. 2007. 177 f. Tese (Doutorado em Patologia Experimental e Comparada) - Universidade de São Paulo, São Paulo, 2007.

ROCHA, C. F. D.; SLUYS, M. V; VTCIBRADIC, D.; KIEFER, M. C.; MENEZES, V. A.; SIQUEIRA, C. C. Comportamento de Termorregulação em Lagartos Brasileiros. Oecologia Brasiliensis, v. 13, p. 115-131, 2009.

ROSENTHAL, K. L. Patologia clínica do iguana verde. In: Bayer Exotics Symposium: Selected Papers on the Green Iguana and Antimicrobials in Exotic Pets, p. 23-26, 2002.

SILVESTRE, A. M.; LAVIN, S. CUENCA, R. La Bioquímica Sanguínea en Clínica de Reptiles. Consulta de Difusión Veterinaria, v. 200, p. 31-40, 2013.

VITT, M. P. L.; MAGNUSSON, W. E.; PIRES, T. C. A.; LIMA, A. P. Guia de Lagartos da Reserva Adolpho Ducke - Amazonia Central. $1^{\text {a }}$ ed. Manaus: Áttema Design Editorial, 2008, 176 p. 